\title{
Virtual Reality Implementation for University Presentation
}

\author{
METIN TURAN \\ Computer Engineering Department \\ İstanbul Commerce University \\ Küçükyalı E5 Kavşağı, İnönü Cad. No:4, Küçükyalı \\ TURKEY \\ https://www.researchgate.net/profile/Metin-Turan-2
}

\begin{abstract}
The purpose of this study is to apply virtual reality technology for university presentation, so that evaluate if it is useful and usable. Due to the pandemic situation, the topic is also relevant to the current situation as many universities have shifted to online classes. With 3D modelling and coding, a university presentation application (only for computer engineering department) with virtual reality base has been developed that will allow the user to navigate around the school building and its surroundings. Because the developed application uses a new technology, it is designed to be convenient and simple considering that it can be difficult to use. Application allows movement in this virtual world according to constraints, as well as providing sound warnings when necessary. The application tested with a helmeted display, represented by Google Cardboard, with two lenses in it and a groove for the phone. The limited experiments by different users showed that the application was impressive, although it was not easy to use devices efficiently for an unexperienced user. On the other hand, with emerging technologies, it is possible develop such applications as web application using Unity WebVR Assests which will make it easy of use.
\end{abstract}

Key-Words: - University Presentation, Virtual Reality, Google Cardboard, Head-Up Display, Unity Programming, 3D Model Design, Google Earth

Received: June 18, 2021. Revised: November 25, 2021. Accepted: December 10, 2021. Published: December 21, 2021.

\section{Introduction}

The concept of Virtual Reality (VR) emerged at the mid of 1960 when Ivan Sutherland first describe VR as a window through which a user perceives the virtual world as real and in which the user could behave realistically [1].

Since it has been applied to different research areas, several definitions have been proposed [2], [3], [4]. These definitions although different, highlight three common features of VR systems: immersion, perception, and interaction with environment [5], [6], [7], [8], [9].

The importance given to VR studies is increasing every day [10], [11], [12]. Thanks to this technology, which is becoming more and more widespread throughout the world, it is possible to access developed software studies on education [13], [14], [15], games [16], [17], three-dimensional promotion [18], [19] and scientific research [20]. A VR experience can be offered with updates to existing software, both to increase marketing capacity and to provide a different experience to the end user [21], [22].

VR can generally be considered as the transfer of real-life scenarios to the computer environment and it is very interwoven with the visual modeling field [23], [24] since modeling is required to use this technology. Any object can be virtualized. The objects, environments, and applications to be modeled are created on a computer environment and are therefore limited to the imagination of the designer [25]. In this context, VR is a computergenerated analogy of a three-dimensional environment where the user experiences and perceives reality [26], [27].

The first example of VR is a ceiling hanging head-mounted device invented by Ivan Sutherland in 1966. 46 years later, in 2012, Palmer Luckey, the founder of the Oculus VR company has presented to the John Carmack, the developer of classic video games such as Doom, Wolfenstein 3D and Quake, a VR device that can be wearable to head. Carmack, who was very impressed with this idea, started an application project with Luckey immediately and this couple presented the DK1 to the world.

These developments attracted the attention of many investors, mainly Mark Zuckerberg, and in 2014, Facebook purchased Oculus for \$ 2 billion. The world's leading companies, such as Google, Sony, Samsung and Steam, have been closely following all these developments, and have begun 
working on this new technology, and VR products have emerged one by one [28].

It can be seen that the areas of application of VR technology can be too many and quite different (from medicine to military use, from entertainment to trade). Nowadays, the most common usage area is education. As a computer-aided education technology, VR is predicted to replace educational technologies and methods used in the future [29].

Scientists in the field of education have developed a wide range of applications. For example, Indrusiak and Reis [30] have transformed the $2 \mathrm{D}$ integrated circuit into a 3D VR model by adding a depth factor to the Web-based VR study they developed. Minogue et al. [31] developed a VR application to show secondary school students the structure and function of animal cells. Another interesting example of VR is the application showing the formation of day and night to 10 -yearold students, using a three-dimensional Earth model with sunbeams, developed by Kerawalla et al. [32]. Murtagh [33] also created a virtual human model for use in Irish Sign Language education with a very useful and comprehensive study. This model has been designed to cover the facial and body movements in order to use the sign language of the deaf individuals in educational analogies.

One of the first comprehensive and successful examples of VR in "Titans in Space" has enabled users to navigate the entire solar system. Science, history, art and mathematics can help students of all ages through VR. Because being able to travel and observe is almost always more fun than reading books to young students.

The topic Augmented Reality (AR) is also a technology associated with VR, operates on the principle of applying computer-generated images (Common Gateway Interface (CGI) to real-world objects. In smart mobile phones, tablets, small console systems such as Nintende 3DS, and even some science museums, CGI can be placed on live video content to create an AR environment. But the biggest distinction between the two is that while the $\mathrm{AR}$ interacts with the components in our environment in the real world, VR working principle is completely software-oriented and closed to the environment. For example, in AR software, we can open our hands and see the trees in our palm, but in the VR software we find ourselves directly in a forest and can trip in the forest. VR and AR software options are expected to emerge in the coming years. Thus, upon request, VR and AR options can be experienced by the user respectively.

Although VR has been applied to the different areas referred above, the motivation of this study is, as far as we know it is the first time, practicing VR technology for university presentation which is relevant in current pandemic situation. An application has been developed that will allow the user (target group is the parents and the candidate students) to perform VR-based navigation around the university school building and its surroundings which are objectified with 3D modelling. Another contribution of this study is to light the way for a researcher how to follow steps to develop such a VR application, briefly it is a detailed technical article for the start-up in this challenging field. The application was used with a helmet display, which was represented by the Google Cardboard, with two lenses and a groove in which the phone was placed. Finally, comments were taken from the experiences of the users and interpreted.

The creation of virtual images in the second section, the technologies used and the merge in the third section, and the results and evaluation in the fourth section of the article are presented.

\section{Material and Methods}

\subsection{Modelling and Scene}

For the SketchUp program, a section of the university building and its surroundings were taken as a screenshot from the Google Earth program and transferred to the program (Fig. 1). These images were modelled by drawing (Fig. 2), so that both a fast and close to a realistic model drawn through this texture led to a better quality of work.

All drawings for building and environment modelling were done in SketchUp. Transparency settings, especially for glass, were easier than tissue I material assignment in Unity. The drawing of the garden view (Fig. 3) and the second floor (Fig. 4) (Computer Engineering Department) were done using Google Earth and the school plan respectively.

Almost all textures, such as benches, trash bins, recycling bins, car models, etc. were obtained from Unity asset store and various Internet sites. Collision detection for each model has been added (mesh collider), thus gaining a walkable model feature.

When the Start object with the Start code in the Main Stage scene is pressed or waited for 3 seconds, the Garden scene is opened, and when passed through the building entrance door (empty and collision) in the Garden stage triggers Change Department code which opens Second Floor scene. Lighting is added to each scene. For the garden scene linear light (a kind of solar function) and for the Second Floor spot light is used. 


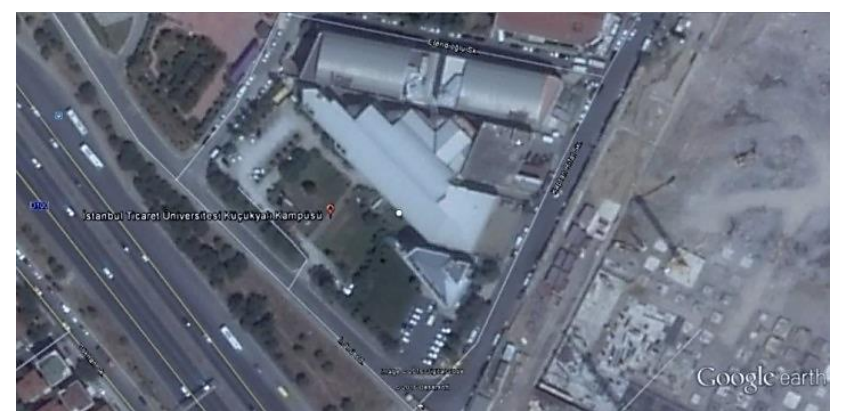

Fig. 1: Google Earth image

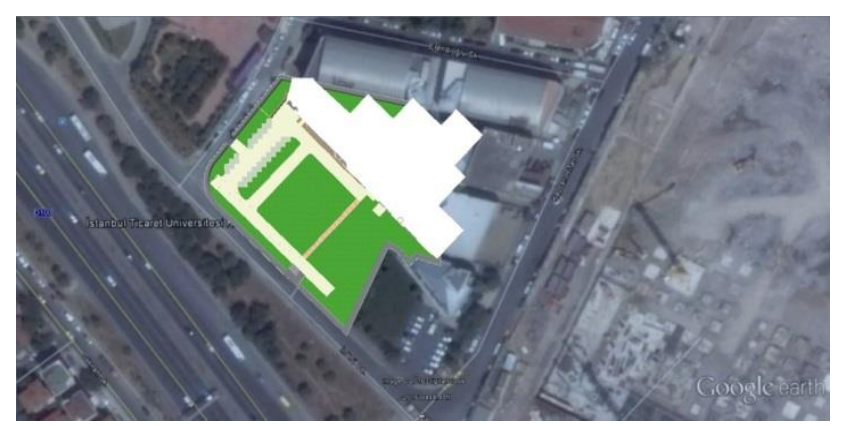

Fig. 2: The model created using Google Earth

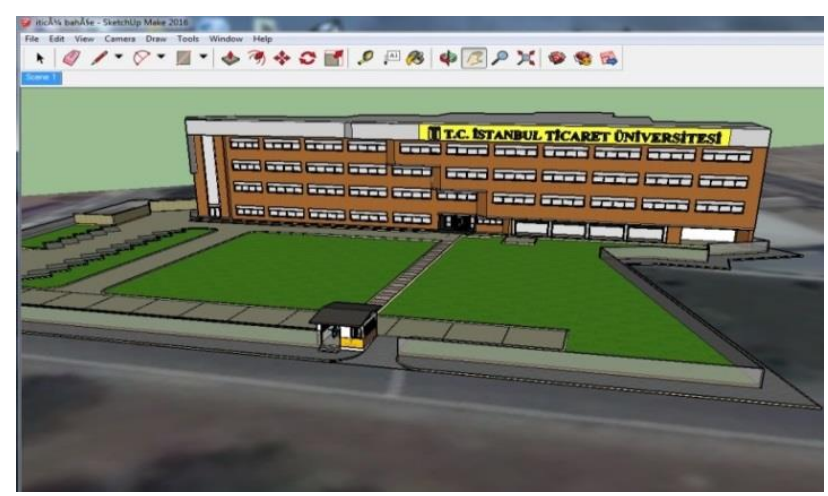

Fig. 3: Garden view of university

\subsection{Used Technologies}

The fact that the user feels that they are in VR and that the world created in the VR arouses sensation of reality in the user is two main concepts sufficient to summarize a VR experience. The maximum experience of VR can take place when the user loses himself / herself in the VR world and cannot discern that it is not in the real world.

In order for the $\mathrm{VR}$ experience to work properly, multiple technologies and techniques come together to form a whole. In general, it can be examined in two areas, as 3D imaging and headmounted monitoring.

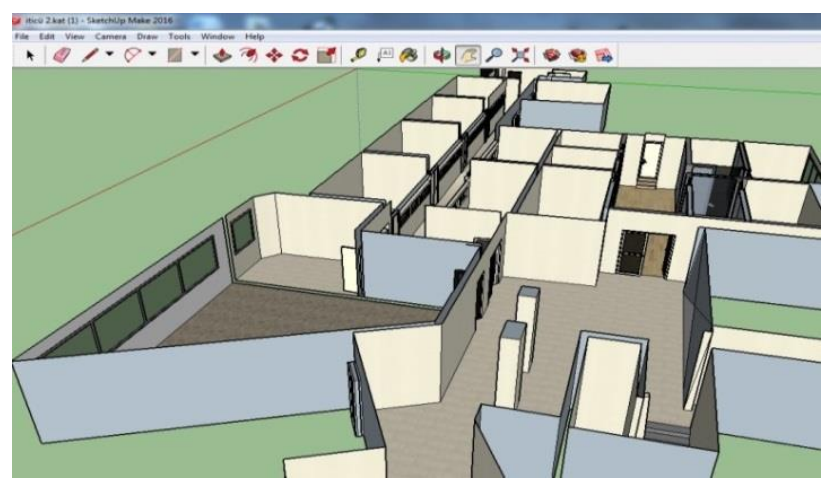

Fig. 4: Second floor model (computer engineering department)

\subsubsection{Google Cardboard}

The Mobile VR represented by Google Cardboard is Head Mounted Display (HMD) with two lenses and a trough in which the phone is placed. The phone's display shows the twin stereographic image. In other words, the angle of view of the left and right eye is adapted to the phone screen. Although there is a rotating head tracking system, no location tracking is available. In addition, the user can touch the screen and make choices within the application.

\subsubsection{Stereoscopic 3D Imaging}

Stereography with split screen appeared shortly before the invention of photography (Fig. 5). Stereo images have two distinct views for the left and right eye, one balanced with respect to the other. Thus, the human brain is perceived as a three-dimensional image.

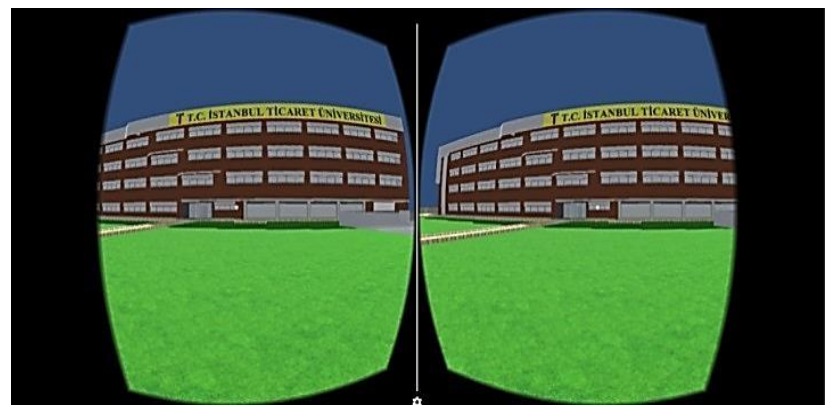

Fig. 5: Screen display without HMD

Likewise, presenting these side-by-side stereo images to users and developers is the first task for VR-enabled cameras in Unity.

When displaying a three-dimensional VR scene, care must be taken to ensure that FPS (frame per second) is not low. If the FPS is too low, the animation (user camera and scene animations) will be intermittent. Factors influencing FPS include GPU performance and the complexity of the scene 
in Unity (many models / animations, polygons, light calculations).

Voice warnings in VR are another important issue. For a complete VR experience, it is important to wear a stereo headset, as it has the effect of increasing the feeling of being in the environment. With VisiSonics (connected to Oculus), which provides the most advanced three-dimensional audio experience, it is ensured that voices can reach the user from the objects in a three-dimensional virtual environment.

\subsubsection{Follow-up}

Inertial Measurement Unit (IMU) can be used to determine the spatial acceleration and rotation values in all three axes. The same sensor is also available in the desktop VR headrest. The current viewpoint is calculated during the head movement with the HMD and is used when drawing the next unit frame. This is called motion detection.

Nowadays, the use of motion detectors is sufficient to play on the phone, but not quite enough for VR. This is due to inadequate / defective calculations, and these defective calculations may eventually accumulate and result in the loss of one's place in the real world. This is one of the biggest disadvantages of Google Cardboard-like mobile VR heads. Head movement can be detected but head position is lost.

Location tracking can be done in VR HMDs of advanced technology. For Oculus Rift, a series of infrared LEDs on the head set are read with an external optical sensor to calculate the user's position.

For Steam VR (HTC Vive), from external to internal position tracking is performed. Two or more low power lasers are operated in the room and read by the optical sensor on the HMD to calculate the user's position.

\subsubsection{Android Developer Studio}

The Android Developer Studio, which hosts the SDK to be integrated into Unity, has been downloaded and installed. After installation, the Android SDK path in Unity is specified.

Since Unity 5.1 has direct VR support, this support is only available for Oculus Rift and Samsung Gear VR HMDs. This work was developed on Google Cardboard and similar HMDs. In contrast, the Google Cardboard offers its own SDK via the Internet and this SDK has been added to Unity's using the Assets tab.

Interacting with software in VR, e.g. keyboard / mouse / click / touch / keystroke, is more a new issue and is examined extensively. The interaction in this project is achieved by one-click or touch and it is very useful for the user. In this context, a file with the codes written in the context of the project was created. A function named Click has been created which controls any mouse button / finger click.

\subsubsection{Unity}

\subsubsection{Student Creation, Path Identification and Execution}

Students should be in and out of the school and these students should move. For this reason, the 3D model Ethan which is ready with its animations was used in Unity. The Third Person Controller prefab in Ethan enables the user to move this character. This prefab in student object (formerly Ethan) was removed because the students would travel around independently from the user. Third Person Controller was added instead. It includes the Navigation Mesh Agent component (which holds the parameters for how the student travels in the virtual world) and the character control code (where the target object is to be traced).

\subsubsection{Interactions: Perspective and Collision}

There are two ways to interact with any object. The first is interaction with the view, and the second is the interaction with the collision.

The interaction with the view also works with the principle of collision. The user can interact when the ray (ray) coming out of the camera collides with another object. The code named Start is created as an example.

This code is added to the start button on the main menu in Unity, which allows the next scene to be loaded when viewed for 3 seconds or when the Cardboard magnet is pulled. Another example is to insert a Card Press object with properties of empty and box collider over the objects passed by pressing the card at the school entrance. When this object is viewed for 3 seconds, or when the Cardboard magnet is pulled, another specified object (here the bars for pass) is hidden and the sound alert is executed. In this way, before the card is read (before interacting with the object), while the door is blocked, when the card is pressed then block is hidden and the user is able to pass.

The interaction with the collision is triggered by the collision of a given object with another object, by excluding the mechanics of the beam described in interaction with the view. For example, an empty metal detector object with a collision box feature is inserted into the metal detector. An audio file and audio code have been added to this object. As a 
result, when this object is passed through, the audio code is running and the detector sound is output accordingly.

\subsubsection{Graphical User Interface}

This layer usually consists of two-dimensional images on the screen. Their intended use is to provide information to the user through status messages, as well as to support interaction by means of visual objects that provide data entry.

The two most common uses are the Head-Up Display (HUD) and the binocular cursor. The HUD is always in sight considered by ignoring the head movement. Mini map, available life information, buttons and components are included in this scope. The binocular cursor is usually located in the center of the screen, allowing the user to better understand which direction the user is looking at and to select objects.

There are 5 additional types of interfaces, but in summary, the separation of these UI techniques depends on where and when they will be used. Within this project, the main menu button and the binoculars cursor (the cursor object is created as a circular shape) has been added to the user screen. Thus, based on the point of view of the cursor, the interaction with the main menu texts and the button on the interface allows the transition to the new scene.

\section{Implementation Details}

\subsection{Character Controls and Their Properties}

In the VR experience, in addition to seeing the world from the first person point of view, the camera must have the head, body and legs just like a real human body. Because by this camera is intended to wander through the virtual world. There are three first-person character components in Unity: camera, rigid body, and capsule collider.

The camera component contains the setting of the viewing parameters of the scene drawn on each updated unit frame. When a camera is added to any object in the scene, that object is defined as a camera object. The stereographic VR camera object provides one view for both eyes. The camera control codes read information such as head position, rotation and direction from the motion sensors of the HMD / mobile device and adjust the camera's conversion values accordingly.

When the rigid body component is added to any object, it is covered by all the calculations in Unity's physics engine. In the rigid body component, gravity, mass, resistance etc. parameters are available. Objects added as solid bodies can collide with each other. For example, two student objects colliding head-on, they will be able to bounce to different directions. If a solid object is added to an object and a camera is added, it will have an object with a physics motor camera. Then, user input parameters can be adjusted, such as running, walking, looking around and jumping.

The encapsulated body is used for collision interaction and character movement, just like a solid object. Although it differs from the rigid body component, it has a physics engine but its rules are not automatically covered. For example, if two students collide head-to-head, they stop at the moment when they collide and do not move like a solid. The biggest advantage of this component is its gravity and earth-related settings. These settings that the height of the stairs, how much rise to stepping up, how much degree will be perceived as a slope, how much slope will give wall effect, generally the force of gravity to be applied to the object are in the encapsulated object. Unless a code is added to this component, the motion is very clear because it does not have a momentum. The object stops directly when it stops, and moves immediately when it moves. The exact opposite of this can be said for the rigid body component, because the motions accommodate momentum, acceleration, and the rules of physics.

There are four character controls with these components:

- Third Person Controller contains the body object in its content. For example, the model used by the student object can be given. This model has a body and animations. When the camera, solid object and encapsulated body are added to this model, a character can be controlled with the contents of the components. There are two codes in the content of Third Person Controller. The first is the Third Person User Control, which controls the user's interaction inputs (keyboard, mouse, click, touch, etc.), and the other Third Person Character adjusts the physical conditions of the user's movements, and controls the animations that are required for running, bending, etc.

- Third Person Controller is the one with the Navigation Mesh Agent and character control codes of Third Person Controller. These two codes allow you to set where and how to move the character, respectively. These controls are used in the student object and movements such as direction finding and progress on the road work in this way. 
- The FPS Controller is a prefabricated unit used for first-person control, which contains both encapsulated body and solid object in addition to the camera. When the character moves, the camera moves with the character object mentioned. By default, the body mass of the object is set to low (1) and the kinematic option is selected. As a result, the momentum property is negligible and the object used does not interact with other solid objects. First Person Controller code inside offers an environment in which many parameters can be set, especially for running, jumping, stepping and sounding at every step.

Finally, the Rigid Body FPS Controller is similar to the FPS Controller that supply first person camera control. It contains a camera and a solid body component but no encapsulated body. When the character moves, the camera moves with the object. The default mass of the Rigid Body FPS Controller is set to 10 and is not kinematic. This means that, in contrast to the FPS Controller, it can be bounced when it collides with another object.

\subsection{First Person Character Control and Setting}

In order to provide an experience close to real life and to avoid abnormal situations in the rules of physics, the first-person character object must be designed to conform to the specific rules within the application.

Some of these rules can be not cut off from the ground when moving or walking, and not pass through the concrete objects or pass by pressing on small objects on that plane which Y axis at different height. Moreover, not to fall from the edge of the created virtual world, and to start and stop with head movement (up and down view) or by user input can be given.

\subsection{Move to Look-Out Direction}

The generated character is considered a camera object due to the presence of a camera. The first thing is to create a code called Head-up Walk. The camera position is advanced in the delta time and at the specified speed in the direction of the Vector3 (used in the jobs that concern the $\mathrm{X}, \mathrm{Y}, \mathrm{Z}$ axes) of this code.

If a person's normal walking speed is considered to be 1.4 meters per second, this speed is reduced to 0.7 , so that it does not come too fast in the VR environment. The Head-up Walk content is controlled by which direction the camera is facing and the position of the character object is moved in this direction (transform). As a result of the tests, the character object was able to move on the $\mathrm{X}, \mathrm{Y}$ and $\mathrm{Z}$ axes. Moving on the $\mathrm{Y}$ axis as an unwanted state causes the character fly. This problem is solved by keeping the foot of the character object on the ground while walking and stopping.

\subsection{Ability to Move through $y$ Axis in Accordance with Rules}

It is unlikely that the $\mathrm{Y}$ axis will be disabled completely, given that the character will step up / down or can walk on the slope. Character is only required to be able to move on the floor. The Y location of constructed model University Garden object (Fig. 3) is at 0. For this reason, it is provided that Head-up Walk is added to the Y axis and can only move at $\mathrm{Y}=0$ height. Since this rule is also applied on all steps in the content of the scene, the character moves on the ground.

\subsection{Not Passing through Concrete Objects}

Another characteristic feature is the addition of a rigid body component to the character object for the rule that character object does not pass through concrete objects. $\mathrm{X}$ and $\mathrm{Z}$ rotations were frozen from the rigid body settings in order to prevent loss of object control after collision. In this way, the edges of the objects are not passed. However, a problem that arises is that in case of collision with other solid objects, the bounce feature of the rigid body is activated. It is not desirable to bounce back from the walls. The solid body component was then removed, instead encapsulated body included in. Thus, in case of collision with other objects as desired, character can be passed by edges and move on the stairs up and down by gravity.

\subsection{Don't Fall from Edge of the Created Scene}

This feature is intended to prevent falling into the infinite space. To accomplish this, the previously mentioned collision detection feature was used. Character object has a collision sensor and cannot pass through the colliding object (unless specifically stated) in the event of a collision. In order to do this, invisible walls with empty and collision features were drawn to the edges of the world. Since character object cannot cross these invisible walls and not fall into the infinite space, it can be safely navigated.

\subsection{Starting and Stopping the Movement}

Acting feature, which is a very important part of this work, and activation / deactivation of this movement 
feature by user input is provided. Within the scope of the study, a code called Click controls whether there is any user input from the keyboard, mouse or Cardboard magnet. By calling the function Click from the Head-up Walk code, the action is made based on the user input.

Finally, by creating the Head Movement code, the movement of the character object is initiated and finished with any user input (click, touch, magnet, etc.) as well as head movement. The feature of the head movement code is that the camera checks to see if its view is below the specified 60 degrees. Thus, only one HMD and a phone can be navigated.

\section{Experiments}

The application was tested by different users (students, teachers, parents) and their opinions were taken (Table 1). The survey gave the following quantitative results:

- $88 \%$ percentage of the users felt the scenes as real. Consequently, VR has potential to be considered alternative technology for university presentation [34].

- $60 \%$ percentage of the users felt it is difficult to use with the HMD. The reason is about the head movements [35]. Consequently, VR devices still need to be enhanced, perhaps with low costs [36].

- $88 \%$ percentage of the users felt over neutral. $40 \%$ percentage so affected via application that users were thrilled during the experience and had a feeling of traveling in another environment. This can be explained by the fascination of a person who has never been dive under water, and the magnificent presence of underwater. Although the user realized that he was in the virtual world, he got enjoyed watching like an immersive film.

We executed another survey additionally on only high-school students (limited to 20 students) these have never seen a university before. We asked if application is useful for generating an idea about universities. Although the application is limited to only one department of the university, $90 \%$ of the students (18 students) took survey gave positive feedback. They considered that it would be useful experience and information about universities in distance away.

\section{Discussions and Conclusion}

In practice, it is aimed to introducing the university with VR model, and evaluate if it is useful and usable. This study, which is carried out only for the computer engineering department, can be applied with the aim of promoting all the university's major attractions. From this perspective, it can be used as a fast and modern application to inform parents on promotional days. However, parents need special support to use the device correctly and efficiently. They can reject to use such devices due to insufficient technological practice experience. On the other hand, with emerging technologies, it is possible develop such applications as web application using Unity WebVR Assests, so that overcoming the need of experience. Moreover, it would be an introducing opportunity for students these are far away from university location for being a student of a university or visiting such as Erasmus program.

Table 1. User experiment results

\begin{tabular}{lllll}
\hline & $\begin{array}{r}\text { Experimenter } \\
\text { (count) }\end{array}$ & $\begin{array}{l}\text { Student } \\
(10)\end{array}$ & $\begin{array}{l}\text { Parent } \\
(10)\end{array}$ & $\begin{array}{l}\text { Teacher } \\
(5)\end{array}$ \\
\hline Question & Options & Answer & Answer & Answer \\
\hline Feel As & Yes & 10 & 8 & 4 \\
Real? & No & 0 & 2 & 1 \\
\hline Easy To & Yes & 6 & 2 & 2 \\
Use? & No & 4 & 8 & 3 \\
\hline What Did & Fascinated & 6 & 3 & 1 \\
You Feel? & Excited & 4 & 5 & 3 \\
& Neutral & 0 & 2 & 1 \\
\hline
\end{tabular}

It is also possible develop such VR applications for other any kind of promotional events such as introduce companies or famous persons to the public. For example, to announce and feature working attractive in a company for talented persons, the facilities in that company can be introduced. A person may introduce himself to the fans by daily activities and/or talents he/she has.

On the other hand, preparing VR applications is laborious and labor-intensive. In another aspect, it requires the production of the script and characters of a game program visually. The preparation of graphics is time consuming and costly. Therefore, although VR applications are exciting, they have a disadvantage in terms of development costs for nonwidespread applications. 
References:

[1] I. E. Sutherland, The Ultimate Display, Multimedia: from Wagner to Virtual Reality, 1965, pp. 506-508.

[2] M. A. Gigante, Virtual Reality: Definitions, History and Applications, Virtual Real. Syst., 1993, pp. 3-14.

[3] H. Fuchs \& G. Bishop, Research Directions in Virtual Environments, Chapel Hill, 1992.

[4] C. Cruz-Neira, Virtual Reality Overview, in SIGGRAPH 93 Course Notes 21 st International Conference on Computer Graphics and Interactive Techniques, 1993, pp. 135-142.

[5] F. Biocca, The Cyborg's Dilemma: Progressive Embodiment in Virtual Environments, Journal of Computer-Mediated Communication, Vol. 3, 1997, pp. 1-29.

[6] J. M. Loomis, J. J. Blascovich \& A. C. Beall, Immersive Virtual Environment Technology as a Basic Research Tool in Psychology, Behavior Research Methods, Instruments \& Computers, Vol. 31, 1999, pp. 557-564.

[7] J. N. Bailenson, N. Yee, D. Merget \& R. Schroeder, The Effect of Behavioral Realism and Form Realism of Real-Time Avatar Faces on Verbal Disclosure, Nonverbal Disclosure, Emotion Recognition and Copresence in Dyadic Interaction, Presence: Teleoperators and Virtual Operators, Vol. 15, 2006, pp. 359372.

[8] M. Slater, Place Illusion and Plausibility can Lead to Realistic Behaviour in Immersive Virtual Environments, Philosophical Transactions of the Royal Society, Vol. 364, 2009, pp. 3549-3557.

[9] S. S. Sundar, Q. Xu \& S. Bellur, Designing Interactivity in Media Interfaces: A Communications Perspective, in Proceedings of the SIGCHI Conference on Human Factors in Computing Systems, 2010, pp. 2247-2256.

[10] M. Vafadar, Virtual Reality: Opportunities and Challenges, International Journal of Modern Engineering Research (IJMER), Vol. 3, 2013, pp. 1139-1145.

[11] M. O. Onyesolu \& F. U. Eze, Chapter 4: Understanding Virtual Reality Technology: Advances and Applications, Advances in Computer Science and Engineering, 2011, pp. 53-71.

[12] S. M. LaValle, Virtual Reality, Cambridge University Press, 2017.

[13] C. Dede, Introduction to Virtual Reality in Education, Themes in Science and Technology Education Special Issue, 2009, pp. 7-9.
[14] V. S. Pantelidis, Reasons to Use Virtual Reality in Education and Training Courses and a Model to Determine When to Use Virtual Reality, Themes in Science and Technology Education Special Issue, 2009, pp. 59-70.

[15] A. Tzanavari \& N. Tsapatsoulis, Affective, Interactive and Cognitive Methods for Elearning, Virtual Reality in Education, 2010, pp. 1-18.

[16] J. Psotka, Educational Games and Virtual Reality as Disruptive Technologies, Educational Technology \& Society, Vol. 16, 2013, pp. 69-80.

[17] A. Sherstyuk, D. Vincent \& A. Treskunov, Towards Virtual Reality Games, in 8th International Conference on Virtual Reality Continuum and its Applications in Industry, 2009, pp. 315-316.

[18] P. Bouvier, F. D. Sorbier, P. Chaudeyrac \& V. Biri, Cross Benefits Between Virtual Reality and Games, in International Conference on Computer Games, Multimedia and Allied Technology, 2008.

[19] C.-S. Chan, Virtual Reality in Architectural Design, Computer Aided Architectural Design Research in Asia Workshops, 1997, pp. 1-10.

[20] P. A. Kopciak, P. Kolar, D. Hellmann, S. El Aeraky \& M. Dollfuss, Virtual Reality Driving Simulator Prototype for Teaching Situational Awareness in Traffic, in Recent Advances in Multimedia Processing, Organization and Visualization beyond Domains and Disciplines, 2016.

[21] L. K. Simone, M. T. Schultheis, J. Rebimbas, \& S. R. Millis, Head-Mounted Displays for Clinical Virtual Reality Applications: Pitfalls in Understanding User Behavior While Using Technology, CyberPsychology \& Behavior, Vol. 9, 2006, pp. 591-602.

[22] J. Mateu, M. J. Lasala \& X. Alamán, Developing Mixed Reality Educational Applications: The Virtual Touch Toolkit, Sensors, Vol. 15, 2015, pp. 21760-21784.

[23] N.-N. Zhou \& Y.-L. Deng, Virtual Reality: A State-of-the-Art Survey, International Journal of Automation and Computing, Vol. 6, 2009, pp. 319-325.

[24] J. Martín-Gutiérrez, C. E. Mora \& B. A.-D. A. González-Marrero, Virtual Technologies Trends in Education, EURASIA Journal of Mathematics Science and Technology Education, Vol. 13, 2017, pp. 469-486.

[25] A. G. Joshi, A. S. Dabhade \& A. S. Borse, Virtual Reality in Android Gaming, International Research Journal of Engineering 
and Technology (IRJET), Vol. 2, 2015, pp. 2322-2327.

[26] S. Mandal, Brief Introduction of Virtual Reality \& Its Challenges, International Journal of Scientific \& Engineering Research, Vol. 4, 2013, pp. 304-309.

[27] M. Zyda, From Visual Simulation to Virtual Reality to Games, Computer, Vol. 38, 2005, pp. 25-32.

[28] J. Linowes, Unity Virtual Reality Projects, Packt Publishing, 2015.

[29] Y. Kayabaşı, Sanal Gerçeklik ve Eğitim Amaçlı Kullanılması, The Turkish Online Journal of Education Technology, Vol. 4, 2005, Article 20.

[30] L. S. Indrusiak \& R. A. Reis, 3D Integrated Circuit Layout Visualization using VRML, Future Generation Computer Systems, Vol. 17, 2001, pp. 503-511.

[31] J. Minogue, M. G. Jones, B. Broadwell \& T. Oppewall, The Impact of Haptic Augmentation on Middle School Students, Conceptions of the Animal Cell, Virtual Reality, Vol. 10, 2006, pp. 293-305.

[32] L. Kerawalla, R. Luckin, S. Seljeflot \& A. Woolard, Making It Real: Exploring the Potential of Augmented Reality for Teaching Primary School Science, Virtual Reality, Vol. 10, 2006, pp. 163-174.

[33] I. Murtagh, Developing a Linguistically Motivated Avatar for Irish Sign Language Visualization, Institute of Technology Blanchardstown Journal, Vol. 10, 2011, pp. 72-70.

[34] H. M. Htike, T. H. Margrain, Y. K. Lai \& P. Eslambolchilar, Ability of Head-Mounted Display Technology to Improve Mobility in People with Low Vision: A Systematic Review, Translational Vision Science \& Technology, Vol. 9, 2020, Article 26.

[35] C.-M. Chang, C.-H. Hsu, C.-F. Hsu \& K.-T. Chen, Performance Measurements of Virtual Reality Systems: Quantifying the Timing and Positioning Accuracy, in Proceedings of the 24th ACM international conference on Multimedia, 2016, pp. 655-659.

[36] B. Lee, C. Yoo, J. Jeong, B. Lee \& K. Bang, Key Issues and Technologies for AR/VR HeadMounted Displays, in Proceedings of SPIE OPTO, Vol. 11304, 2020.

\section{Creative Commons Attribution License 4.0 (Attribution 4.0 International, CC BY 4.0)}

This article is published under the terms of the Creative Commons Attribution License 4.0 https://creativecommons.org/licenses/by/4.0/deed.en _US 